\title{
Structural changes in metallic nanoislands on 2D materials during in situ annealing in ultra-high vacuum TEM
}

\author{
Kate Reidy ${ }^{1}$, Joachim Dahl Thomsen ${ }^{1}$, Vera Zarubin ${ }^{1}$ and Frances Ross ${ }^{2}$ \\ ${ }^{1}$ Massachusetts Institute of Technology (MIT), United States, ${ }^{2}$ MIT, United States
}

The effect of high temperature on crystalline metal structures plays an important role in the understanding of many physical and chemical phenomena, from wetting, to catalysis, coarsening, and diffusion [13]. Many of these processes are driven by surface energy minimization and are a function of both the crystallographic facets of the metal present, and the interface energy with the substrate [4]. Moreover, the surrounding gaseous environment also plays a role: the coarsening of nanoporous gold [5], the initial stages of solid-state dewetting of $\mathrm{Au}$ films [6], and the faceting of Pt catalysts [7] are significantly influenced by surface adsorbates at elevated temperature.

Most of these studies have been carried out on bulk $\mathrm{Si}$ or $\mathrm{SiO}_{2}$ substrates as these are especially relevant to current technological applications [8,9]. However, with the growing adoption of two-dimensional (2D) materials in electronic and opto-electronic devices, characterisation of the annealing interaction between a 2D material and a bulk 3D metal is paramount for 2D device integration at elevated temperature. Due to the inherent van der Waals bonding of the 2D material, the interface energies and consequently the phenomena of nucleation, growth, epitaxy, diffusion, dewetting, and agglomeration can be markedly different to what is observed on conventional covalently bonded and amorphous substrates [10-14].

Here, we investigate the effect of temperature on the growth, morphology, dewetting, and agglomeration of single crystal metallic nanoislands on 2D materials. Our sample preparation utilises ultra-high vacuum (UHV) metal deposition on suspended 2D material substrates (Figure 1a). The UHV deposition chamber is connected to a Hitachi H-9000 UHV TEM to allow imaging without breaking vacuum, as well as recording movies during in-situ heating and gas flow. Nanoislands deposited on van der Waals bonded surfaces of 2D materials can grow epitaxially to be crystallographically aligned with the underlying 2D material substrate. For several metals, at low temperature growth conditions we observe dendritic shapes of nanoislands, whereas high temperatures sees a characteristic transition from dendritic to single crystalline, facetted nanoislands (Figure 1b-g). This phenomena is explored for Au as a model system, as well as other technologically relevant materials, $\mathrm{Ti}$ and $\mathrm{Nb}$.

We then use in-situ UHV TEM to compare the island morphology obtained via post-growth annealing to that obtained via heating during metal deposition. Dewetting and agglomeration behaviour of these single crystalline nanoislands is imaged via resistive heating in-situ in the UHV TEM. At low temperatures, we observe dewetting and sintering of Au nanoislands that possess coalescence boundaries or unfaceted side edges (Figure 2). However, single crystalline facetted islands of triangular shape are remarkably stable up to several hundred ${ }^{\circ} \mathrm{C}$, which we speculate is due to their closeness to the equilibrium Winterbottom shape. We find a large substrate dependence of the dewetting and agglomeration behaviour. Islands on 2D materials agglomerate to form large coalesced areas whereas those on $\mathrm{SiN}$ form small isolated spheres.

Subsequently, we compare the morphology and dewetting behaviour of these single crystalline and facetted islands on 2D materials under conditions of oxygen flow in the UHV TEM. Here, adsorbed oxygen affects diffusion on the 2D material substrate as well as self-diffusion across the nanoisland surface. Together, these results inform mechanisms and temperature dependence on the processing of 3D 
metallic nanoislands grown on 2D material substrates, as well as showcasing the effect of gaseous environment on the reaction [15].
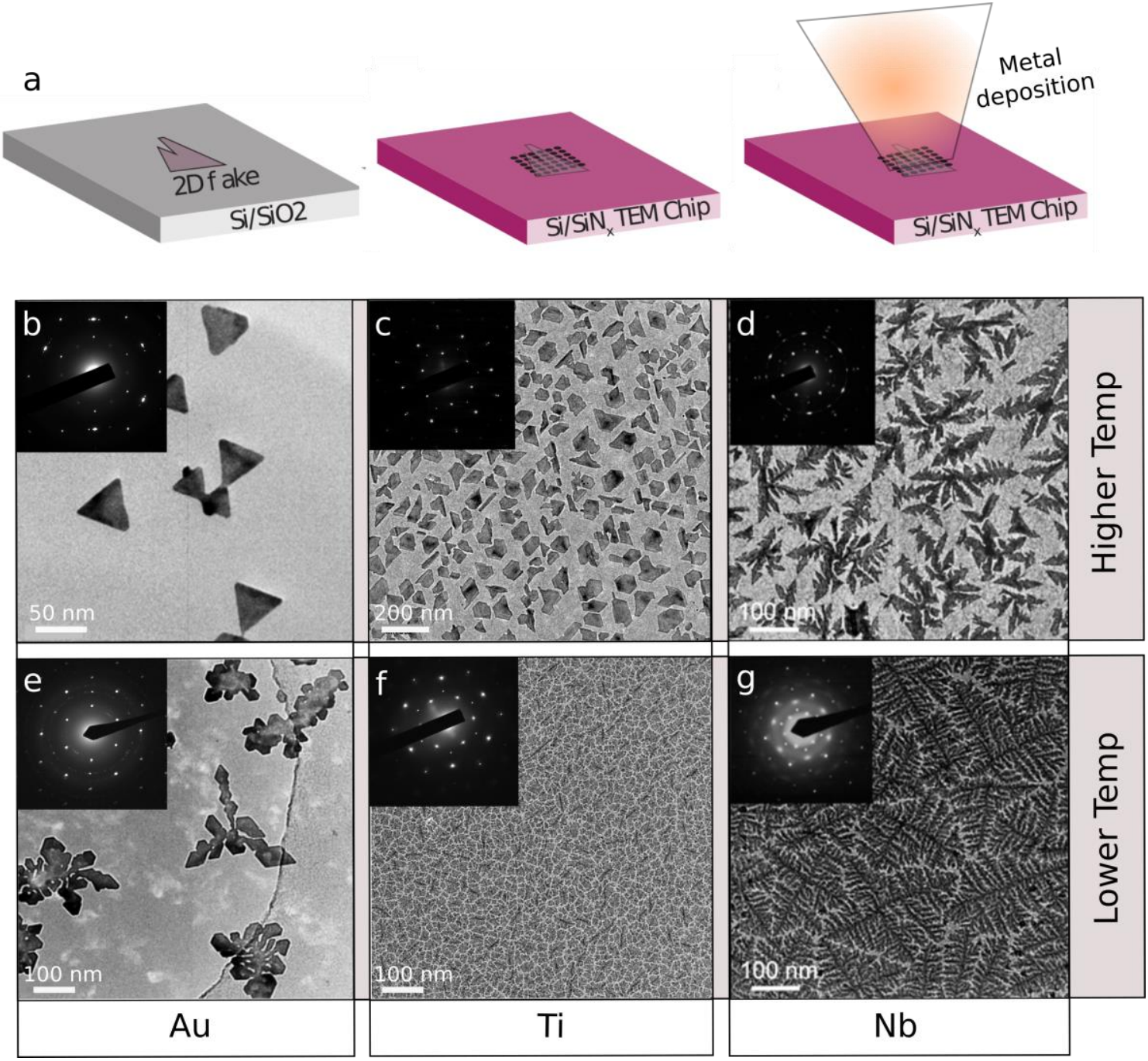

Figure 1. Dependence of temperature on crystal growth of $\mathrm{Au}, \mathrm{Ti}$, and $\mathrm{Nb}$. a) Schematic of transfer process to create suspended 2D materials, on which metals can be deposited under ultra-high vacuum. $b$ - d) Facetted deposition of b) $\mathrm{Au}, \mathrm{c}$ ) $\mathrm{Ti}$, and d) $\mathrm{Nb}$ nanoislands on graphene at high temperature, and e f) dendritic deposition of e) $\mathrm{Au}, \mathrm{f}$ ) $\mathrm{Ti}$, and g) $\mathrm{Nb}$ nanoislands on graphene at low temperature. Note the definition of 'high' and 'low' temperature depends on the melting temperature of the material. 

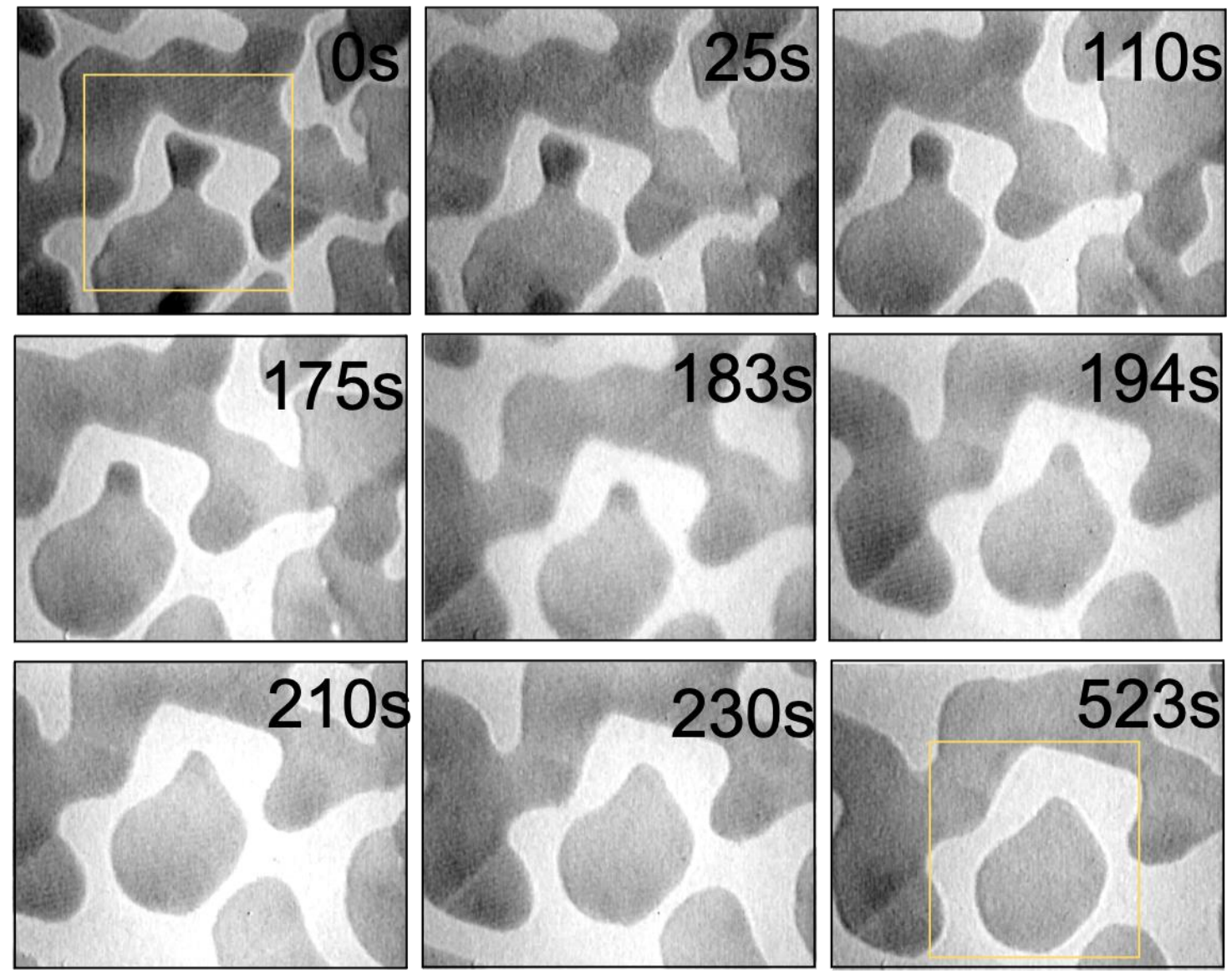

Figure 2. In-situ heating of Au on MoS2. a) Movie stills of 0 - 523 seconds of Au on MoS2 in-situ heating in UHV TEM at $\sim 200^{\circ} \mathrm{C}$. Dewetting and sintering observed.

\section{References}

[1] Balluffi, R. W. J. Nucl. Mater. 69, 240-263 (1978).

[2] Niether, C., et al. Nat. Energy 3, 476-483 (2018).

[3] Thompson, C. V. Annu. Rev. Mater. Res. 42, 399-434 (2012).

[4] Giermann, A. L. \& Thompson, C. V. J. Appl. Phys. 109, 1-8 (2011).

[5] Chen, A. Y., et al. Appl. Surf. Sci. 355, 133-138 (2015).

[6] Kosinova, A., et al. Acta Mater. 83, 91-101 (2015).

[7] Harris, P. J. F. Nature 323, 792-794 (1986).

[8] Seguini, G., et al. Nanotechnology 25, 495603 (2014).

[9] Batič, B. Š., et al. Vacuum 138, 134-138 (2017).

[10] Somers, L. A., et al. Phys. Rev. B 82, 115430 (2010).

[11] Luo, Z., et al. Nano Lett. 10, 777-781 (2010).

[12] Liu, L., et al. J. Phys. Chem. B 117, 4305-4312 (2013).

[13] Lee, S. H., et al. Appl. Phys. A Mater. Sci. Process. 118, 389-396 (2014).

[14] Reidy, K., et al. Nat Commun 12, 1290 (2021) 
[15] This work made use of facilities and instrumentation supported by NSF through the Massachusetts Institute of Technology Materials Research Science and Engineering Center DMR - 1419807, as well as facilities at MIT.nano. K.R. acknowledges funding from an OGE MIT Fellowship. J.D.T acknowledges support from Independent Research Fund Denmark though grant number 9035-00006B. V.Z. acknowledges funding from an MSA Undergraduate Research Scholarship. 